\title{
Pricing in Noncooperative Interference Channels for Improved Energy-Efficiency
}

\author{
Rami Mochaourab, Zhijiat Chong, and Eduard Jorswieck \\ Communications Theory, Communications Laboratory \\ Dresden University of Technology, Dresden, Germany \\ e-mail: \{Mochaourab, Chong, Jorswieck\}@ifn.et.tu-dresden.de
}

\begin{abstract}
We consider noncooperative energy-efficient resource allocation in the interference channel. Energy-efficiency is achieved when each system pays a price proportional to its allocated transmit power. In noncooperative game-theoretic notation, the power allocation chosen by the systems corresponds to the Nash equilibrium. We study the existence and characterize the uniqueness of this equilibrium. Afterwards, pricing to achieve energy-efficiency is examined. We introduce an arbitrator who determines the prices that satisfy minimum QoS requirements and minimize total power consumption. This energy-efficient assignment problem is formulated and solved. Simulation results on energy-efficiency are then given where we compare our setting to the one without pricing. It is observed that pricing in this distributed setting achieves higher energy-efficiency in different interference regimes.
\end{abstract}

\section{INTRODUCTION}

Power management and energy-efficient communication is an important topic in future mobile communications and computing systems. Currently, $0.14 \%$ of the carbon emissions are contributed by the mobile telecommunications industry [1]. In order to improve the situation, we study new algorithms at physical and multiple access layers. This includes resource allocation and power allocation. A common mobile communication scenario is where several communication system pairs utilize the same frequencies and are within interference range from one another. This setting is modeled by the interference channel (IFC). The transmit-receiver pairs could belong to different operators and these are not necessarily connected. Therefore, noncooperative operation of the systems is assumed.

In a noncooperative scenario without pricing, systems transmit at highest possible powers to maximize their data rates. Transmitting at high powers however is detrimental to other users, because it induces interference which reduces their data rates. In such settings, spectrum sharing might lead to suboptimal operating points or equilibria [2]. The case of distributed resource allocation and the conflicts in noncooperative spectrum sharing are best analyzed using noncooperative game theory (e.g. for CDMA uplink in [3] and usage of auction mechanisms in [4]). An overview of power control using game theory is presented in [5]. Moreover, analysis of

This work is supported in part by the Deutsche Forschungsgemeinschaft (DFG) under grant Jo 801/4-1 and by the German Federal State of Saxony in the Excellence Cluster Cool Silicon in the framework of the project Cool Cellular under grant number 14056/2367. noncooperative and cooperative settings using game theory are performed in [6].

Studies have shown that the point of equilibrium in a noncooperative game is inefficient but can be improved by introducing a linear pricing [7]. Linear pricing means that each system has to pay an amount proportional to the power it uses to transmit. It is observed that this encourages transmission at lower powers, which reduces the amount of interference and at the same time leads to a Pareto improvement in the users' payoffs. Pricing in multiple-access channels has also been investigated with respect to energy-efficiency in [8].

In [9] the energy-efficiency of point-to-point communication systems is improved by sophisticated adaptation strategies. A coding theoretic approach is proposed in [10] where "green codes" for energy-efficient short-range communications are developed. Recent proposals define a utility function which incorporates the cost of transmission, e.g., the price of spending power is considered in a binary variable in [11] and as an inverse factor in [12].

A similar utility function as in this paper is proposed in [13] for single-antenna systems and used to characterize the Nash equilibrium for the noncooperative power control game. Later in [14], the approach is extended to multiple antenna channels in a related noncooperative game-theoretic setting. In [15], distributed pricing is introduced for power control and beamforming design to improve sum rate.

Different from previous works, we apply linear pricing to improve the energy-efficiency of an IFC with noncooperative selfish links to enable distributed implementation. Our objectives also include global stability and fairness. Instead of proving the uniqueness of the Nash Equilibrium (NE), as in [3], we derive a set of prices in which the uniqueness is guaranteed, which is then utilized as a constraint in the optimization problem. The contribution is the derivation of the optimal pricing for transmit power minimization under minimum utility requirements and spectrum sharing constraints.

This paper is organized as follows. In Section II, the system, channel and the game models are presented. The game described is then studied in Section III. Based on uniqueness analysis of the Nash equilibrium, we formulate and solve the energy-efficient optimization problem with minimum utility requirements constraint in Section IV. In Section V, simulations comparing the setting with and without pricing are presented. Section VI concludes this paper. 


\section{PRELIMINARIES}

\section{A. System Model}

Two wireless links communicate on the same frequency band at the same time. Transmitter $T_{i}$ intends to transmit its signal to its corresponding receiver $R_{i}, i \in\{1,2\}$. On simultaneous transmission, each receiver receives a superposition of the signals transmitted from both transmitters. Assuming single-user decoding, the interfering signal is treated as additive noise. This system model can be extended to multiple system pairs. For convenience, we focus our analysis on two pairs.

The described competing links belong to different operators or wireless service providers. We assume that there exists an entity which can control the operators indirectly by rules or by changing their utility functions. We could think of this entity as a national or international regulatory body. In contrast to common long term regulation, the utility function here changes on a smaller time-scale. In section II-C, the role of the arbitrator which represents this authority is discussed.

\section{B. Channel Model}

We consider a quasi-static block-flat fading IFC in standard form [16]. The direct channel coefficients are unity. The cross channel coefficients (CCC), which are the squared amplitudes of the channel gains, from $T_{i}$ to $R_{j}$ are denoted as $\alpha_{i j}$. The noise at the receivers is independent additive white Gaussian with variance $\sigma^{2}$. The inverse noise power is denoted by $\rho$, i.e. $\rho=1 / \sigma^{2}$. The transmitters and receivers are assumed to have perfect channel state information (CSI). The maximum achievable rate at receiver $R_{1}$, analogously $R_{2}$, is written as

$$
R_{1}\left(p_{1}, p_{2}\right)=\log _{2}\left(1+\frac{\rho p_{1}}{1+\rho \alpha_{21} p_{2}}\right)
$$

where $p_{i}, i \in\{1,2\}$, is the transmit power of $T_{i}$. We assume no power constraint on the transmitters, i.e. $p_{i} \in \mathbb{R}^{+}$. It is shown later that the maximum power that would be utilized is nevertheless bounded due to a pricing factor.

\section{Game Model}

A game in strategic form consists of a set of players, a set of strategies that each player chooses from, and the payoffs which each player receives on application of a certain strategy profile. The players of our game are the communication links and are denoted by the corresponding subscript. The pure strategy of each player $i, i \in\{1,2\}$, is the transmission power $p_{i}$. The corresponding payoff is expressed in the utility function

$$
u_{i}\left(p_{1}, p_{2}\right)=R_{i}\left(p_{1}, p_{2}\right)-\mu_{i} p_{i}, \quad i=1,2,
$$

where $R_{i}\left(p_{1}, p_{2}\right)$ is given in (1) and $\mu_{i}>0$ is the power price for player $i$. The second term in (2) is a pricing term, which linearly reduces the utility. This means that a payment is demanded from the player for the amount of power used. Without pricing, each user would use as much power as possible to transmit his signal [17]. The game is written as $G=\left(\{1,2\},\left(\mathbb{R}^{+}, \mathbb{R}^{+}\right),\left\{u_{1}, u_{2}\right\}\right)$. We assume all players are rational and individually choose their strategies to maximize their utilities. The game is assumed to be static, which means that each player decides for one strategy once and for all. The outcome of this game is a Nash equilibrium (NE). A NE is a strategy profile $\left(p_{1}^{\mathrm{NE}}, p_{2}^{\mathrm{NE}}\right)$ in which no player can unilaterally increase his payoff by deviating from his NE strategy, i.e. for player $1, u_{1}\left(p_{1}^{\mathrm{NE}}, p_{2}^{\mathrm{NE}}\right) \geq u_{1}\left(p_{1}, p_{2}^{\mathrm{NE}}\right)$ for all $p_{1} \in \mathbb{R}^{+}$, and similarly for player 2 .

The best response, $b r_{i}$, of a player $i$ is the strategy or set of strategies that maximize his utility function for a given strategy of the other player. Since the player's utility function is concave in his own strategy, the best response is unique and given as the solution of the first derivative being zero. The best response for player 1 is written as

$$
b r_{1}\left(p_{2}\right)=\left(\frac{1}{\mu_{1}}-\frac{1}{\rho}-\alpha_{21} p_{2}\right)^{+}, \quad p_{2} \in \mathbb{R}^{+},
$$

where $(x)^{+}$denotes $\max (x, 0)$. The The highest power a transmitter $T_{i}$ may allocate is given as $p_{i}^{\max }=b r_{i}(0)=$ $\left(\frac{1}{\mu_{i}}-\frac{1}{\rho}\right)^{+}$, which is achieved when $p_{j}=0$ for $j \neq i$. Thus, the strategy region of player $i$ could be confined to $\left[0, p_{i}^{\max }\right]$.

The authority that can control the elements of the game is assumed to determine the power prices, $\mu_{1}$ and $\mu_{2}$. In game-theoretic notation, this entity is called the arbitrator [18]. The arbitrator is not a player in the game, and chooses the equilibrium that meets certain criteria. In our case these criteria would be fairness, energy-efficiency and minimum utility requirements. We assume that the arbitrator also has complete game information.

\section{NONCOOPERATIVE GAME}

In this section we study the game described in Section II-C. This is done by investigating the existence of pure strategy NEs and characterizing the conditions for uniqueness.

\section{A. Existence of Nash Equilibrium}

There exists a pure strategy NE in a game if the following two conditions are satisfied [19]. First, the strategy spaces of the players should be nonempty compact convex subsets of an Euclidean space. Second, the utility functions of the players should be continuous in the strategies of all players and quasiconcave in the strategy of the corresponding player.

The first condition is satisfied in our game because the strategy space of player $i$ is $\left[0, p_{i}^{\max }\right] \subset \mathbb{R}$. The second condition is satisfied for the following reasons. First, it is obvious that the utility functions are continuous in the players' strategies. Second, knowing that all concave functions are quasi-concave functions [20], we can prove the concavity of our utility function with respect to the corresponding player's strategy by showing that $\frac{\partial^{2} u_{i}\left(p_{1}, p_{2}\right)}{\partial p_{i}^{2}}<0$. Next, we analyze the number of NEs that exist and state the related conditions.

\section{B. Uniqueness of Nash Equilibrium}

In this section, we study the conditions that lead to a unique NE. Considering only the case where simultaneous utilization of the spectrum is made, we prove that the best response dynamics there globally converge. Under these conditions, the 
noncooperative systems are guaranteed to operate in the NE if they iteratively apply their best response strategies.

Proposition 1: There exists a unique NE if and only if the following condition is satisfied

$$
\begin{array}{r}
{\left[\alpha_{12}<\frac{\mu_{1}\left(\rho-\mu_{2}\right)}{\mu_{2}\left(\rho-\mu_{1}\right)}\right]} \\
\text { or }\left[\alpha_{21}<\frac{\mu_{2}\left(\rho-\mu_{1}\right)}{\mu_{1}\left(\rho-\mu_{2}\right)}\right] .
\end{array}
$$

Proof: The proof is given in [21].

Following the conditions in (4), we can easily characterize the sufficient conditions for the existence of a unique NE.

Corollary 1: There exists a unique NE if $\alpha_{12} \alpha_{21}<1$.

If the conditions in (4a) and (4b) are fulfilled simultaneously, both transmitters would be transmitting at the same time. We denote this case as the concurrent transmission case. Next, we consider only this case since it is the case where both systems operate simultaneously, hence a fair assignment. The other cases in which a unique NE exists correspond to one transmitter allocating maximum transmit power and the other not transmitting. The concurrent transmission case satisfies $\alpha_{12} \alpha_{21}<1$, which is the sufficient condition for the existence of a unique NE given in Corollary 1.

In the concurrent transmission case, the transmitters operate in the unique $\mathrm{NE}$ which is a fixed point of the best response function. It is necessary in order to reach the NE that the best response dynamics globally converge.

Proposition 2: The best response dynamics globally converge to the $\mathrm{NE}$ in the concurrent transmission case, i.e. when (4a) and (4b) hold simultaneously.

Proof: The proof is given in [21].

In comparison to the IFC without pricing, the sufficient conditions for global convergence of the best response dynamics are identical. The reason for that is however not obvious. The linear pricing in our utility function leads to a translation of the best response function but as well changes the interference conditions where concurrent transmission takes place. This is seen in the conditions in (4a) and (4b) where the bounds are dependent on the prices. Therefore, proving the sufficient conditions for global convergence of the best response dynamics is necessary in our case.

\section{Admissible Power Prices}

Given $\alpha_{12}, \alpha_{21}$, and $\rho$, there exists a set of pricing pairs that achieves the concurrent transmission case described above. We define an admissible power pricing set $\mathcal{M}$ as

$$
\mathcal{M} \triangleq\left\{\begin{aligned}
\left(\mu_{1}, \mu_{2}\right): \quad 0<\mu_{1}<\rho, \\
\mu_{2}<\hat{\mu}_{2}\left(\mu_{1}\right)=\frac{\frac{1}{\alpha_{12}} \rho \mu_{1}}{\rho-\mu_{1}\left(1-\frac{1}{\alpha_{12}}\right)}, \\
\mu_{2}>\check{\mu}_{2}\left(\mu_{1}\right)=\frac{\alpha_{21} \rho \mu_{1}}{\rho-\mu_{1}\left(1-\alpha_{21}\right)}
\end{aligned}\right\} .
$$

All $\left(\mu_{1}, \mu_{2}\right) \in \mathcal{M}$ achieve NEs in the concurrent transmission case. In the case that $\alpha_{12} \alpha_{21}>1$, the set $\mathcal{M}$ is however empty, i.e. there exists no power prices that achieve the concurrent transmission case. This happens since the upper bound on $\mu_{2}$ would be less than the lower bound for any $\mu_{1}$, i.e. $\hat{\mu}_{2}\left(\mu_{1}\right)<\check{\mu}_{2}\left(\mu_{1}\right)$. Another observation is that the set $\mathcal{M}$ is convex only in the case if $\alpha_{12}<1$ and $\alpha_{21}<1$ both hold. This corresponds to the weak interference case. In the case if one CCC is larger than one, but still the condition $\alpha_{12} \alpha_{21}<1$ holds, the set $\mathcal{M}$ is not convex. We call this the strong interference case.

The unique NE in the concurrent transmission case as a function of the power prices is calculated as

$$
p_{1}^{\mathrm{NE}}\left(\mu_{1}, \mu_{2}\right)=\frac{1}{1-\alpha_{12} \alpha_{21}}\left(\frac{1}{\mu_{2}}-\frac{1}{\rho}-\frac{\alpha_{12}}{\mu_{1}}+\frac{\alpha_{12}}{\rho}\right),
$$

and $p_{2}^{\mathrm{NE}}\left(\mu_{1}, \mu_{2}\right)$ is given by swapping the indices 1 and 2 in (6), where $\left(\mu_{1}, \mu_{2}\right) \in \mathcal{M}$.

\section{ENERGy-EFFicient AssignMEnt}

In this section, we investigate how the power prices are chosen such that energy-efficiency as well as minimum utility requirements are satisfied.

\section{A. Optimization problem}

The arbitrator needs to know which power prices $\left(\mu_{1}, \mu_{2}\right)$ he should choose such that the outcome satisfies the following conditions.

C1 The best response dynamics globally converge to the unique NE.

C2 Spectrum sharing (concurrent transmission) is ensured so that it is fair for all users.

C3 Users transmit at the lowest powers possible but still satisfying minimum utility requirement $u_{i}^{\mathrm{r}}, i \in\{1,2\}$, to promote efficient energy usage.

If $\left(\mu_{1}, \mu_{2}\right) \in \mathcal{M}$, conditions $\mathrm{C} 1$ and $\mathrm{C} 2$ are automatically fulfilled. Condition C3 can be achieved by optimization. Hence, determining the optimal prices $\left(\mu_{1}^{*}, \mu_{2}^{*}\right)$ is done by solving the following programming problem

$$
\begin{gathered}
\min _{\left(\mu_{1}, \mu_{2}\right)} P\left(\mu_{1}, \mu_{2}\right) \\
\text { s.t. } \quad u_{i}\left(p_{1}^{\mathrm{NE}}, p_{2}^{\mathrm{NE}}\right) \geq u_{i}^{\mathrm{r}}, \quad i=1,2, \\
\left(\mu_{1}, \mu_{2}\right) \in \mathcal{M} .
\end{gathered}
$$

The objective function is calculated as

$$
\begin{aligned}
& P\left(\mu_{1}, \mu_{2}\right)=p_{1}^{\mathrm{NE}}\left(\mu_{1}, \mu_{2}\right)+p_{2}^{\mathrm{NE}}\left(\mu_{1}, \mu_{2}\right) \\
& =\frac{\left(1-\alpha_{12}\right)}{\mu_{1}\left(1-\alpha_{12} \alpha_{21}\right)}+\frac{\left(1-\alpha_{21}\right)}{\mu_{2}\left(1-\alpha_{12} \alpha_{21}\right)}-\frac{2-\alpha_{12}-\alpha_{21}}{\rho\left(1-\alpha_{12} \alpha_{21}\right)} .
\end{aligned}
$$

The function in (8) is convex in $\left(\mu_{1}, \mu_{2}\right)$ only in the weak interference channel case, i.e. $\alpha_{12}, \alpha_{21}<1$. Similarly, the constraint set $\mathcal{M}$ is also only convex in the weak interference channel case. Thus, the problem in (7) is in general not a convex optimization problem. Next, we consider only the case of weak interference channel.

\section{B. Weak interference channel}

For the condition that $\alpha_{12}$ and $\alpha_{21}$ are both less than one, we provide a closed form solution to the problem in (7). 
Definition 1 (Dominating vector): A vector $\left(\mu_{1}, \mu_{2}\right)$ is said to dominate vector $\left(\nu_{1}, \nu_{2}\right)$ if $\mu_{i} \geq \nu_{i}$ for all $i$ s and $\mu_{i}>\nu_{i}$ for at least one component.

Using this definition, we study the monotonicity properties of the sum power in NE function given in (8) with respect to the vector $\left(\mu_{1}, \mu_{2}\right)$.

Lemma 1: The objective function (8) is monotonically decreasing in $\left(\mu_{1}, \mu_{2}\right)$ with respect to the order prescribed by Definition 1.

Proof: The changes of $P\left(\mu_{1}, \mu_{2}\right)$ with respect to prices $\mu_{1}$ and $\mu_{2}$ are observed to be negative in their derivatives, such that

$$
\frac{\partial P\left(\mu_{1}, \mu_{2}\right)}{\partial \mu_{1}}=-\frac{1-\alpha_{12}}{\left(1-\alpha_{12} \alpha_{21}\right) \mu_{1}^{2}}<0
$$

and similarly with respect to $\mu_{2}$. Thus, any increment in $\mu_{1}$ or $\mu_{2}$ reduces $P\left(\mu_{1}, \mu_{2}\right)$. This implies that for any series of vector $\left(\mu_{1}^{i}, \mu_{2}^{i}\right)$ where $\left(\mu_{1}^{i}, \mu_{2}^{i}\right)$ dominates $\left(\mu_{1}^{j}, \mu_{2}^{j}\right)$ and $i>j$, $P\left(\mu_{1}^{i}, \mu_{2}^{i}\right)<P\left(\mu_{1}^{j}, \mu_{2}^{j}\right)$.

The optimal power prices corresponds to the vector that dominates according to Definition 1 all vectors that satisfy the minimum utility requirement constraints.

Proposition 3: The optimal power prices $\left(\mu_{1}^{*}, \mu_{2}^{*}\right)$ which solve programming problem (7) are given as

$$
\begin{aligned}
& \mu_{1}^{*}=\frac{g\left(u_{1}^{\mathrm{r}}\right) g\left(u_{2}^{\mathrm{r}}\right)-\frac{g\left(u_{1}^{\mathrm{r}}\right) g\left(u_{2}^{\mathrm{r}}\right)}{\alpha_{12} \alpha_{21}}+g\left(u_{1}^{\mathrm{r}}\right)+g\left(u_{2}^{\mathrm{r}}\right)+1}{\frac{1}{\rho \alpha_{12} \alpha_{21}}\left(\left(1-\alpha_{21}\right) g\left(u_{2}^{\mathrm{r}}\right)-\alpha_{21}\right)}, \\
& \mu_{2}^{*}=\frac{g\left(u_{1}^{\mathrm{r}}\right) g\left(u_{2}^{\mathrm{r}}\right)-\frac{g\left(u_{1}^{\mathrm{r}}\right) g\left(u_{2}^{\mathrm{r}}\right)}{\alpha_{12} \alpha_{21}}+g\left(u_{1}^{\mathrm{r}}\right)+g\left(u_{2}^{\mathrm{r}}\right)+1}{\frac{1}{\rho \alpha_{12} \alpha_{21}}\left(\left(1-\alpha_{12}\right) g\left(u_{1}^{\mathrm{r}}\right)-\alpha_{12}\right)},
\end{aligned}
$$

where $g(x) \triangleq \frac{W\left(-2^{-1-x} \log (2)\right)}{\log (2)}$ and $W(\cdot)$ is the Lambert W function ${ }^{1}$.

Proof: The proof is given in [21].

Remark 1: In the strong interference case, one of the CCCs is greater than one. However, the condition $\alpha_{12} \alpha_{21}<1$ should hold in order for the admissible power prices set $\mathcal{M}$ not to be empty. In this interference regime, we can show that the solution given in Proposition 3 also solves the programming problem given in (7).

\section{Feasible Minimum Utility Requirements}

Given minimum utility requirements, $u_{1}^{r}$ and $u_{2}^{r}$, the arbitrator should be able to determine if this pair is feasible, i.e. whether there exists a power pricing pair $\left(\mu_{1}, \mu_{2}\right)$ that fulfill these requirements simultaneously.

Proposition 4: A minimum utility requirement $\left(u_{1}^{r}, u_{2}^{r}\right)$ is feasible if and only if the optimal power prices $\left(\mu_{1}^{*}, \mu_{2}^{*}\right)$ calculated in (10) and (11) are in the admissible power prices set $\mathcal{M}$ given in (5), i.e. $\left(\mu_{1}^{*}, \mu_{2}^{*}\right) \in \mathcal{M}$.

Proof: The proof is given in [21].

Therefore, according to Proposition 4, the arbitrator checks if $\left(\mu_{1}^{*}, \mu_{2}^{*}\right) \in \mathcal{M}$ in order to determine the feasibility of the minimum utility requirements.

In the next section, we give numerical simulations on energy-efficiency comparing the noncooperative setting with

\footnotetext{
${ }^{1}$ The Lambert $\mathrm{W}$ function satisfies $W(z) e^{W(z)}=z[22]$.
}

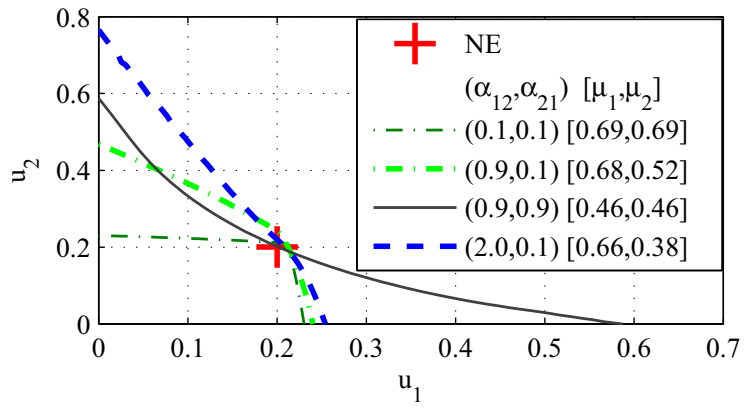

Fig. 1. The Pareto boundaries for various $\left(\alpha_{12}, \alpha_{21}\right)$ as shown in round brackets in the legend, $\left(u_{1}^{r}, u_{2}^{r}\right)=(0.2,0.2)$ and $\rho=10 \mathrm{~dB}$. The corresponding optimal prices as shown in square brackets. The NE and $\left(u_{1}^{r}, u_{2}^{r}\right)$ are in the identical position.

pricing with that without pricing as well as the cooperative setting with pricing.

\section{Simulations}

The Pareto boundaries for various $\left(\alpha_{12}, \alpha_{21}\right)$ pairs are plotted in Fig. 1 for the noncooperative case with pricing. It shows the utility regions that are feasible, given $\left(\alpha_{12}, \alpha_{21}\right),\left(u_{1}^{r}, u_{2}^{r}\right)$, $\rho$ and the corresponding optimal power prices $\left(\mu_{1}^{*}, \mu_{2}^{*}\right)$. This was done by first obtaining points in the utility region by computing $\left(u_{1}, u_{2}\right)$ according to (2) by varying the powers $p_{1}$ and $p_{2}$, where $p_{1} \in\left[0, p_{1}^{\max }\right]$ and $p_{2} \in\left[0, p_{2}^{\max }\right]$, and then grouping the scattered points into equally spaced bins in the $u_{1}$ axis. Using the points with the highest $u_{2}$ for every bin, the Pareto boundary is plotted. Changing only $\rho$ does not have any effect on the Pareto boundaries or the NE. Practically, the operating points along the Pareto boundary are achievable when the systems cooperate.

As expected, the NE in the utility region, which is calculated by inserting $\left(p_{1}^{\mathrm{NE}}\left(\mu_{1}^{*}, \mu_{2}^{*}\right), p_{2}^{\mathrm{NE}}\left(\mu_{1}^{*}, \mu_{2}^{*}\right)\right)$ into (2), is found exactly at the utility requirements, independently of the values $\left(\alpha_{12}, \alpha_{21}\right)$. The NE is very close to the Pareto boundaries, indicating that it is indeed a very Pareto-efficient operating point for various CCCs. By increasing $\alpha_{12}=\alpha_{21}$ simultaneously, the utility region is expanded in that the intersections at the $u_{1}$ and $u_{2}$ axes increase. The region is also observed to change from being convex to being nonconvex as the product $\alpha_{12} \alpha_{21}$ becomes larger. The reason for this is that prices are reduced so that systems can reach the utility requirements at higher CCCs. This also implies that cooperation among systems is more advantageous to achieve a higher sum utility than noncooperation when the potential interference is high.

With regard to the optimal prices, which is shown in the legend of Fig. 1, we observe that the system with the smaller CCC has to pay less than the one with the larger. However, if both systems have large CCCs, both pay less.

An appropriate metric for comparing energy-efficiency is defined as

$$
E=\frac{\sum_{i=1,2} R_{i}}{\sum_{i=1,2} p_{i}} \text { (bits/Joule), }
$$



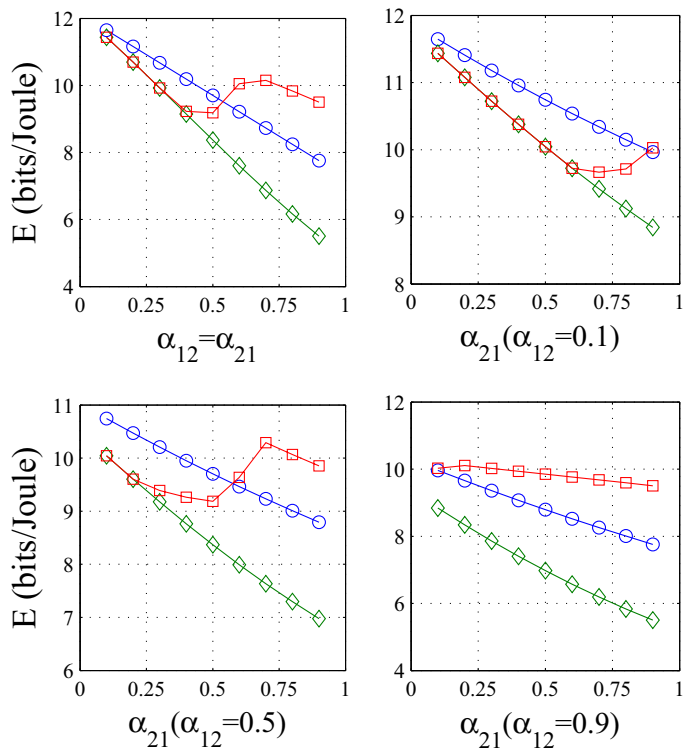

Fig. 2. Comparison of energy-efficiency $E$ with various CCCs. The noncooperative case with pricing (S1) is plotted with blue circles, the noncooperative case without pricing (S2) with green diamonds, and the cooperative case with pricing (S3) with red squares.

where $R_{i}$ is the transmission rate, as in (1), of system $i$ and $p_{i}$ the corresponding power allocation. Fig. 2 shows a comparison between energy-efficiency in the following settings.

S1 The NE achieved with pricing.

S2 The NE achieved without pricing.

S3 Both systems cooperatively choose their strategies to achieve the highest sum utility, i.e. $u_{1}+u_{2}$.

The operating point for the cooperative case was determined numerically.

We see that in the noncooperative case, pricing improves the energy-efficiency significantly. The amount of improvement increases as the CCCs increase. The results with cooperation prove to be superior when the CCCs are large, whereas for low CCCs, noncooperation with pricing yields better energyefficiency. One might expect the outcome of cooperation to be always superior to that of noncooperation. This is not true here because in the case of cooperation, the sum utility is maximized instead of $E$. In our scenario, systems are only interested in maximizing their sum utility but not energyefficiency when cooperating.

\section{CONCLUSIONS}

In this work, we consider two communication system pairs that operate in a distributed manner in the same spectral band. In order to improve the system energy-efficiency, we employ linear pricing to the utility of the systems. Following that, we study the setting from a noncooperative game-theoretic perspective, i.e. we analyze the existence and uniqueness of the Nash equilibrium. Based on the assumption that there exists an arbitrator that chooses the power prices, we considered the problem of minimizing the sum transmit power with the constraint of satisfying minimum utility requirements. We derived an analytical solution for the optimal power prices that solve this problem. Simulation results show that the noncooperative operating points with pricing are always more energy-efficient than that without pricing. A further extension of this work is to consider the case with more than two users.

\section{REFERENCES}

[1] "Sustainable energy use in mobile communications," ERICSSON White Paper, Tech. Rep., 2007.

[2] R. Etkin, A. Parekh, and D. Tse, "Spectrum sharing for unlicensed bands," IEEE Journal on Selected Areas in Communications, vol. 25, no. 3, pp. 517-528, Apr. 2007.

[3] T. Alpcan, T. Basar, R. Srikant, and E. Altman, "CDMA Uplink Power Control as a Noncooperative Game," Wireless Networks, vol. 8, no. 6, pp. $659-670,2002$.

[4] J. Huang, R. A. Berry, and M. L. Honig, "Auction-Based Spectrum Sharing," Mobile Networks and Applications, vol. 11, no. 3, pp. 405408, 2006.

[5] M. Chiang, P. Hande, T. Lan, and C. W. Tan, "Power control in wireless cellular networks," Found. Trends Netw., vol. 2, no. 4, pp. 381-533, 2008.

[6] A. Leshem and E. Zehavi, "Game theory and the frequency selective interference channel," Signal Processing Magazine, IEEE, vol. 26, no. 5, pp. 28-40, Sept. 2009

[7] C. Saraydar, N. Mandayam, and D. Goodman, "Efficient power control via pricing in wireless data networks," Communications, IEEE Transactions on, vol. 50, pp. 291-303, Feb. 2002.

[8] E. Jorswieck and H. Boche, "Energy-aware utility regions: Multiple access pareto boundary," in Communications, 2009. ICC '09. IEEE International Conference on, June 2009, pp. 1-5.

[9] B. Bougard, G. Lenoir, A. Dejonghe, L. V. Der Perre, F. Catthoor, and W. Dehaene, "SmartMIMO: An energy-aware adaptive MIMO-OFDM radio link control for next-generation wireless local area networks," EURASIP JWCN, pp. Article ID 98 186, 2007.

[10] P. Grover and A. Sahai, "Green codes: Energy-efficient short-range communication," Prof. of IEEE ISIT, pp. 1178-1182, 2008.

[11] D. Acemoglu, A. Ozdaglar, and R. Srikant, "The marginal user principle for resource allocation in wireless networks," IEEE Conf. on Dec. and Control, vol. 2, pp. 1544-1549, 2004.

[12] F. Meshkati, M. Chiang, H. V. Poor, and S. C. Schwartz, "A gametheoretic approach to energy-efficient power control in multicarrier cdma systems," IEEE Journal on Selected Areas in Communications, vol. 24, pp. 1115-1129, 2006.

[13] S. Gunturi and F. Paganini, "Game theoretic approach to power control cellular CDMA," Proc. of IEEE VTC, 2003.

[14] J. Duplicy, J. Louveaux, and L. Vandendorpe, "Utility-based MIMO uplink beamforming," Proc. IEEE Workshop SAMSP, pp. 254-257, 2006.

[15] D. Schmidt, C. Shi, R. Berry, M. Honig, and W. Utschick, "Distributed resource allocation schemes," Signal Processing Magazine, IEEE, vol. 26, no. 5, pp. 53-63, Sept. 2009.

[16] A. Carleial, "Interference channels," Information Theory, IEEE Transactions on, vol. 24, no. 1, pp. 60-70, Jan. 1978.

[17] E. Larsson and E. Jorswieck, "Competition versus cooperation on the MISO interference channel," IEEE Journal on Selected Areas in Communications, vol. 26, no. 7, pp. 1059-1069, Sept. 2008.

[18] R. B. Myerson, "An introduction to game theory," no. 623, Sept. 1984

[19] J. B. Rosen, "Existence and uniqueness of equilibrium points for concave n-person games," Econometrica, vol. 33, no. 3, pp. 520-534, 1965.

[20] S. Boyd and L. Vandenberghe, Convex Optimization. Cambridge University Press, Mar. 2004.

[21] Z. Chong, R. Mochaourab, and E. Jorswieck, "Pricing in noncooperative interference channels for improved energy-efficiency," submitted to EURASIP JWCN. [Online]. Available: http://nt.wcms-file2.tudresden.de/BibtexDbMng/upload/Chong2010wen.pdf

[22] R. M. Corless, G. H. Gonnet, D. E. G. Hare, D. J. Jeffrey, and D. E. Knuth, "On the Lambert W function," Adv. Comput. Math, vol. 5, p. 329, 1996. 